\title{
SUNstudy Study Protocol: Sarcoma Usual versus Negative Pressure Study Protocol
}

\section{David Shelds}

Department of Orthopaedic Oncology, Glasgow Royal Infirmary, Castle Street, Glasgow, UK

Corresponding author: David Shields, Department of Orthopaedic Oncology, Glasgow Royal Infirmary, Castle Street, Glasgow, UK, E-mail: dwshields@gmail.com

Received date: November 06, 2017; Accepted date: November 20, 2017; Published date: November 27, 2017

Citation: David Shields (2017) SUNstudy Study Protocol: Sarcoma Usual versus Negative pressure dressing Study Protocol. J Clin Exp Orthop Vol.3 No.3:47

Copyright: (02017 Shield D. This is an open-access article distributed under the terms of the Creative Commons Attribution License, which permits unrestricted use, distribution, and reproduction in any medium, provided the original author and source are credited.

\section{Abstract}

Background: Tumour excision with negative margins is the ultimate goal following soft tissue sarcoma excision, but obtaining this goal can come at a cost with high wound complication rates reported. There is however an emerging body of evidence that primary application of a negative pressure dressing can significantly decrease the risk of developing post-operative wound complications. This paper describes the study protocol for an interventional randomized controlled trial, comparing the efficacy of negative pressure dressings against standard dressings on primary closed wounds following soft tissue sarcoma excision.

Questions/Purposes: Does primary application of negative pressure dressing to closed wounds post soft tissue sarcoma excision reduce wound complications when compared to standard dressings?

Level of evidence: Level I, Prospective RCT

Keywords: Sarcoma; Negative pressure dressing; Control trial; Wound management

\section{Introduction}

\section{Background}

Soft tissue sarcomas are rare tumours of mesenchymal cell origin representing under $1 \%$ of all adult tumours in the United Kingdom [1]. Principles of treatment have evolved over the last decade from primary amputation to limb preservation, with tumour resection and negative margins the ultimate goal [2]. Ensuring clear margins, however, can come at a cost. Large wounds with a disrupted vascular supply and lymphatic drainage may result in increased wound complications such as infection, dehiscence and delayed wound healing $[3,4]$. Wound problems remain one of the most common complications following resection of a soft tissue sarcoma and represent a major source of post-operative morbidity. Peat et al. report wound complication rates of $16 \%$ [4], however Baldini et al. suggest rates as high as 56\%, especially seen in those who have undergone neo-adjuvant radiotherapy or with margins close to the skin surface [5]. In the last 15 years there is an emerging body of evidence suggesting a reduction in wound complications with the primary application of a negative pressure dressing $[6,7,8,9-11]$. However, the negative pressure dressings typically have an increased cost and added complexity in usage compared to conventional dressings, meaning that their routine use remains elusive. Within the high-risk group of patients undergoing resection of a soft tissue sarcoma there is little evidence of the beneficial use of negative pressure dressings. A recent case-controlled study performed at our unit, however, demonstrates encouraging results with zero surgical site complications in patients who underwent sarcoma resection followed by primary wound closure and application of a negative pressure dressing [8].

\section{Rationale}

The aim of this trial is to compare the effectiveness of negative pressure dressing on closed wounds following soft tissue sarcoma excision. Our null hypothesis is there will be no difference in surgical site infections (as defined by the Health Protection Agency) between wounds covered by negative pressure dressings and those covered in standard dressings following soft tissue sarcoma excision.

\section{Study question}

Does primary application of negative pressure dressing to closed wounds post soft tissue sarcoma excision reduce wound complications when compared to standard dressings?

\section{Methods}

\section{Study design and setting}

This is an interventional prospective randomized control trial, comparing the efficacy of negative pressure dressings against standard dressings on primary closed wounds following soft 
tissue sarcoma excision. Patients will be initially recruited from the West of Scotland Regional Sarcoma Service based at Glasgow Royal Infirmary, NHS Greater Glasgow and Clyde. Our aim however is to expand recruitment to other specialist centers.

\section{Participants/study subjects}

Patients will be identified by Orthopedic Musculoskeletal (MSK) Oncology specialists at the time of diagnosis and when the decision for operative intervention has been planned. Eligibility for inclusion will be made by the principle investigator, or a suitably qualified delegated MSK specialist.

\section{Inclusion criteria}

- Diagnosis of soft tissue sarcoma on the upper limb, lower limb, or torso

- Consented for wide local excision or planned marginal excision of the tumour

- Age 16-85 years inclusive

- English speaking

\section{Exclusion criteria}

- Previous surgery to planned surgical field

- Contra-indication to surgical excision of the tumour

- Primary preferred management via amputation

- Disseminated malignancy on pre-operative radiological imaging

- Post-radiation sarcoma

- Allergy to adhesive dressing

- Current psychological condition that will affect compliance to follow up

- Patients refusal for inclusion

\section{Consent procedures}

Patients are fully informed and consent obtained prior to inclusion in this study. All patients will be provided with a patient information sheet and have the opportunity to discuss the trial with both an impartial expert and the principle investigator at their first orthopaedic appointment. The patient will be given at least $24 \mathrm{~h}$ to review the information sheet and digest information given to them prior to obtaining full written informed consent at a subsequent clinic appointment prior to surgery. All patients will be provided with a direct phone number to an oncology nurse specialist who is highly experienced in sarcoma medical and surgical management, but also independent from the study data collection and analysis. The process of consent will be in accordance with the Declaration of Helsinki (2013).

\section{Description of experiment and treatment}

Study Group 1: Standard dressing. The soft tissue sarcoma will be excised as a wide local excision or planned marginal excision as per the pre-operative plan. The wound will be closed in layers as per standard surgical technique with skin closure as per the operating surgeon's preference with the absence of skin glue. Wounds will be covered in a conventional absorbent transparent dressing. Low-molecular weight heparin thromboprophylaxis will be provided to all patients post-operatively until discharge as per local protocol.

Study Group 2: Negative pressure dressing. The soft tissue sarcoma will be excised as a wide local excision or planned marginal excision as per the pre-operative plan. The wound will be closed in layers as per standard surgical technique with skin closure as per the operating surgeon's preference with the absence of skin glue. A mesh based dressing will be applied to the wound following by a transparent occlusive dressing as per the manufacturer's instruction. The dressing will then be connected to a suction device (ActiV.A.C. ${ }^{\circledR}, \mathrm{KCl}$ ), providing a constant negative pressure of $120 \mathrm{mmHg}$. The negative pressure dressing will be left in-situ for 5-6 days as per the manufactures instructions. Low-molecular weight heparin thromboprophylaxis will be provided to all patients post-operatively until discharge as per local protocol.

\section{Aftercare}

All patients will have the same standardized post-operative wound management including intravenous antibiotics until surgical drains are removed. Dressings will be clinically assessed daily until discharge by the operating team or by a designated specialist nurse as appropriate.

\section{Description of followup routine}

Outpatient follow-up will be pragmatic depending on postoperative wound healing with standardized follow-up planned for all patients at 30 days post-operatively. An adverse event will be defined as any untoward medical occurrence that the patient experiences whilst participating in the study. These will be immediately reported to the Principle Investigator and recorded in the patient's records as per standard policy.

\section{Variables and outcome measures}

\section{Primary Outcome:}

Any surgical site infection (SSI) as defined by the Health Protection Agency by day 30 post-operatively [12]

\section{Secondary Outcomes:}

- Wound dehiscence or further surgery to the operative site by day 30 post-operatively

- Time to wound dryness-day post-operative where there has been $12 \mathrm{~h}$ with no further wetness on the dressing or no further volume in VAC unit (days)

- Surgical drain duration and volume ( $\mathrm{ml})$

- Duration of hospital inpatient stay (days)

- Functional assessments

1. TESS (Toronto Extremity Salvage Score) [13]

2. MSTS (Musculoskeletal Tumour Society) score at 3, 6, and 12 months post-operatively [14].

- Adverse events-Any unanticipated events (morbidity, mortality, reaction of dressing) 


\section{Data collection}

Baseline data will be collected from both paper notes and electronic patient databases (Bluespeir, Worcestershire, UK). Data will be anonymised and collated on Excel (Microsoft $\subset$, USA) by a blinded researcher. Baseline data will include: Age, gender, weight, height, ASA grade, smoking status, significant medical history, diagnosis [15], any intra-operative complication, drain volume, length of surgery, length of hospital stay, grade of operating surgeon, and any adjuvant chemotherapy or radiotherapy within the 30 -day post-operative period.

Primary outcome of surgical site infection and secondary outcomes of wound dehiscence or further surgery will be recorded at any time point of inpatient or outpatient follow-up up to 30 days post-operatively. Time to wound dryness, as defined by no new staining on the wound dressing in a $12 \mathrm{~h}$ period, will be made by a specialist oncology nurse during the patients inpatient stay. Functional assessments will be made pre-operatively and post-operatively at 3, 6, and 12 months post-operatively.

\section{Ethical approval}

Ethical approval has been obtained via the West of Scotland Research Ethical Committee-(approval number 16/WS/0146) as part of the national Integrated Research Application System.

\section{Statistical analysis, study size}

We have estimated current SSI rate to be $20 \%$ following excision of a soft tissue sarcoma and standard wound dressing based on the literature [4] and our internal outcome data. A case-control trial performed at our unit demonstrated zero SSI's after negative dressing application in a cohort of 9 patients who underwent sarcoma excision [8]. We propose a clinically significant improvement in SSI rate to be $15 \%$, thereby aiming for an overall SSI rate of $5 \%$. Power calculation for the primary outcome with a $95 \%$ confidence interval and power set to 0.80 the sample size was calculated at 77 patients per arm. Allowing for $10 \%$ loss to follow-up, we aim to recruit 85 patients per arm. If a patient declines or was unable to continue in their assigned group they will be analysed in their assigned group as per the intention to treat concept.

\section{Results}

Publication and dissemination of findings to medical practitioners, patients, and key stakeholders will be undertaken through a number of methods:

- The study protocol and findings will be submitted for publication in peer-reviewed journals

- Findings will be presented at national and international meetings. These will include both general orthopedic and specialist musculoskeletal oncology conferences.

\section{Discussion}

This paper describes the study protocol 2.7 (14th Sept 2016) for the SUNstudy, which has been fully approved by ethics. Our intention is for expansion to multicentres with recruitment starting at our site in the near future.

\section{Randomized Controlled Trial (RCT) Details}

Registry Name: www.clinicaltrials.gov

Registration Number: NCT02901405

\section{References}

1. Paul AS, Charalambous JA, Maltby B, Whitehouse R (2003) The management of soft-tissue sarcomas of the extremity. Current Orthopaedics 17: 124-133.

2. Grimer R, Judson I, Peake D, Seddon B (2010) Guidelines for the management of soft tissue sarcomas. Sarcoma 15: 506182.

3. Moore J, Isler M, Barry J, Mottard S (2014) Major wound complications risk factors following soft tissue sarcoma resection. Eur J Surg Oncol 40: 1671-1676.

4. Peat BG, Bell RS, Davis A, O'Sullivan B, Mahoney J, et al. (1994) Wound-healing complications after soft-tissue sarcoma surgery. Plast Reconstr Surg 93: 980-987.

5. Baldini EH, Lapidus MR, Wang Q, Manola J, Orgill DP, et al. (2013) Predictors for major wound complications following pre-operative radiotherapy and surgery for soft tissue sarcoma of the extremity and trunk: importance of tumor proximity to the skin surface. Ann Surg Oncol 20: 1494-1499.

6. Hansen E, Durinka JB, Costanzo JA, Austin MS, Deirmengian GK (2013) Negative pressure wound therapy is associated with resolution of incisional drainage in most wounds after hip arthroplasty. Clin Orthop Relat Res 47: 3230-3236.

7. Karlakki S, Brem M, Giannini S, Khanduja V, Stannard J, et al. (2013) Negative pressure wound therapy for management of the surgical incision in orthopaedic surgery. A review of evidence and mechanisms for an emerging indication. Bone Joint Res 2: 276-84.

8. Kong R, Shields D, Bailey O, Gupta S, Mahendra A (2017) Negative pressure wound therapy for closed surgical wounds in musculoskeletal oncology patients-A case-control trial. Open Orthop J 11: 502-507.

9. Reddix RN, Leng XI, Woodall J, Jackson B, Dedmond B, et al. (2010) The effect of incisional negative pressure therapy on wound complications after acetabular fracture surgery. J Surg Orthop Adv 19: 91-97.

10. Stannard JP, Robinson JT, Anderson ER, McGwin G, Volgas DA, et al. (2006) Negative pressure wound therapy to treat hematomas and surgical incisions following high-energy trauma. J Trauma 60: 1301-1306.

11. Stannard JP, Volgas DA, McGwin G, Stewart RL, Obremskey W, et al. (2012) Incisional negative pressure wound therapy after highrisk lower extremity fractures. J Orthop Trauma 26: 37-42.

12. Harrington P, Wloch C, Elgohari S, Lamagni T (2013) Protocol for the Surveillance of Surgical Site Infection. Surgical Site Infection Surveillance Service. London: Public Heath England.

13. Davis AM, Wright JG, Williams JI, Bombardier C, Griffin A, et al (1996) Development of a measure of physical function for patients with bone and soft tissue sarcoma. Qual Life Res 5: 508-516.

14. Enneking WF, Dunham W, Gebhardt MC, Malawar M, Pritchard DJ (1993) A system for the functional evaluation of reconstructive 
procedures after surgical treatment of tumors of the musculoskeletal system. Clin Orthop Relat Res 286: 241-246.
15. Fletcher C, Unni K, \& Mertens F (2002) World Health Organization Classification of Tumours. Lyon: IARC Press. 